\title{
Prior Infarcts, Reactivity, and Angiography in Moyamoya Disease (PIRAMD): a scoring system for moyamoya severity based on multimodal hemodynamic imaging
}

\author{
Travis R. Ladner, BA, ${ }^{1}$ Manus J. Donahue, PhD, ${ }^{1}$ Daniel F. Arteaga, BA, ${ }^{1}$ Carlos C. Faraco, PhD, ${ }^{1}$ \\ Brent A. Roach, MD, ${ }^{1}$ L. Taylor Davis, MD, ${ }^{1}$ Lori C. Jordan, MD, PhD, ${ }^{2}$ \\ Michael T. Froehler, MD, PhD, ${ }^{3}$ and Megan K. Strother, MD ${ }^{1}$
}

'Department of Radiology; '2Division of Pediatric Neurology, Department of Pediatrics; and ${ }^{3}$ Departments of Neurology and Neurosurgery, Vanderbilt University Medical Center, Nashville, Tennessee

\begin{abstract}
OBJECTIVE Quantification of the severity of vasculopathy and its impact on parenchymal hemodynamics is a necessary prerequisite for informing management decisions and evaluating intervention response in patients with moyamoya. The authors performed digital subtraction angiography and noninvasive structural and hemodynamic MRI, and they outline a new classification system for patients with moyamoya that they have named Prior Infarcts, Reactivity, and Angiography in Moyamoya Disease (PIRAMD).

METHODS Healthy control volunteers $(n=11$; age $46 \pm 12$ years [mean \pm SD]) and patients $(n=25 ; 42 \pm 13.5$ years) with angiographically confirmed moyamoya provided informed consent and underwent structural (T1-weighted, T2weighted, FLAIR, MR angiography) and hemodynamic (T2*- and cerebral blood flow-weighted) 3-T MRI. Cerebrovascular reactivity (CVR) in the internal carotid artery territory was assessed using susceptibility-weighted MRI during a hypercapnic stimulus. Only hemispheres without prior revascularization were assessed. Each hemisphere was considered symptomatic if localizing signs were present on neurological examination and/or there was a history of transient ischemic attack with symptoms referable to that hemisphere. The PIRAMD factor weighting versus symptomatology was optimized using binary logistic regression and receiver operating characteristic curve analysis with bootstrapping. The PIRAMD finding was scored from 0 to 10. For each hemisphere, 1 point was assigned for prior infarct, 3 points for reduced CVR, 3 points for a modified Suzuki Score $\geq$ Grade II, and 3 points for flow impairment in $\geq 2$ of 7 predefined vascular territories. Hemispheres were divided into 3 severity grades based on total PIRAMD score, as follows: Grade 1, 0-5 points; Grade 2, 6-9 points; and Grade 3, 10 points.
\end{abstract}

RESULTS In 28 of $46(60.9 \%)$ hemispheres the findings met clinical symptomatic criteria. With decreased CVR, the odds ratio of having a symptomatic hemisphere was $13(95 \% \mathrm{Cl} 1.1-22.6, \mathrm{p}=0.002)$. The area under the curve for individual PIRAMD factors was $0.67-0.72$, and for the PIRAMD grade it was 0.845 . There were $0 / 8(0 \%), 10 / 18(55.6 \%)$, and 18/20 (90\%) symptomatic PIRAMD Grade 1, 2, and 3 hemispheres, respectively.

CONCLUSIONS A scoring system for total impairment is proposed that uses noninvasive MRI parameters. This scoring system correlates with symptomatology and may provide a measure of hemodynamic severity in moyamoya, which could be used for guiding management decisions and evaluating intervention response.

http://thejns.org/doi/abs/10.3171/2015.11.JNS15562

KEY WORDS moyamoya; cerebrovascular reactivity; magnetic resonance imaging; hemodynamic; vascular disorders

$\mathrm{M}$ OYAMOYA is a progressive stenoocclusive disease of the distal internal carotid arteries (ICAs) and their proximal terminal branches. Patients most frequently present with ischemic stroke or recurrent tran- sient ischemic attacks. Quantification of the severity of vasculopathy and its impact on vascular morphology and parenchymal hemodynamics is a necessary prerequisite for informing management decisions and evaluating inter-

ABBREVIATIONS ASPECTS = Alberta Stroke Program Early CT Score; AUC = area under the curve; BOLD = blood oxygen level-dependent; $\mathrm{CBF}=$ cerebral blood flow; $\mathrm{CBV}=$ cerebral blood volume; $\mathrm{CVR}=$ cerebrovascular reactivity; $\mathrm{DSA}=$ digital subtraction angiography; $\mathrm{ETCO}_{2}=$ end-tidal $\mathrm{CO}_{2} ; \mathrm{ICA}=$ internal carotid artery; $\mathrm{MCA}=$ middle cerebral artery; $\mathrm{mSS}$ = modified Suzuki score; PIRAMD = Prior Infarcts, Reactivity, and Angiography in Moyamoya Disease; ROC = receiver operating characteristic; Xe-CT = xenon-enhanced CT.

SUBMITTED March 10, 2015. ACCEPTED November 3, 2015.

INCLUDE WHEN CITING Published online March 11, 2016; DOI: 10.3171/2015.11.JNS15562. 
vention response. Current management decisions use angiographic data combined with MRI evidence of infarct, along with perfusion studies based on PET or SPECT. Studies of hemodynamic reserves are probably a useful adjunct; however, current clinical methods require exogenous contrast administration and/or ionizing radiation. Although such tools have improved our understanding of moyamoya, these methods are suboptimal for longitudinal monitoring of patients or assessing revascularization response, because of dose restrictions.

Cerebrovascular reactivity (CVR) is a well-documented and valuable surrogate marker of cerebrovascular reserve in patients with previously identified intravascular pathology. ${ }^{1,9-12}$ In healthy parenchyma, CVR primarily derives from a large increase in cerebral blood flow $(\mathrm{CBF})$ and cerebral blood volume (CBV) in response to a vasostimulatory agent such as $\mathrm{CO}_{2}$ (i.e., hypercapnia). Significantly diminished or negative changes in CVR during hypercapnia have previously been shown to correlate with regions affected by prior infarct and symptomatology. ${ }^{1,26,29}$ However, due to the relative novelty of hypercapnic CVR mapping performed using MRI compared with more established clinical measures such as acetazolamide SPECT, interpretation of hypercapnic CVR maps has not been standardized.

In this study we outline an integrated, neuroimagingbased classification system for moyamoya severity that we have named Prior Infarcts, Reactivity, and Angiography in Moyamoya Disease (PIRAMD). This scoring system accounts for functional measurements of hemodynamic impairment in moyamoya by using blood oxygen leveldependent (BOLD) MRI-weighted CVR, and therefore may be a comprehensive approach toward stratification of moyamoya severity.

\section{Methods \\ Patient Selection}

All procedures were followed in accordance with the ethical standards of the Vanderbilt University Institutional Review Board. Patients presenting with angiographically confirmed moyamoya between January 2011 and May 2015 underwent hemodynamic 3-T MRI (Philips) performed using body coil transmission and 12-channel sensitivity-encoding (SENSE) array neurovascular coil for reception. Patients were included in the study if cerebral digital subtraction angiography (DSA) was performed within 90 days of MRI, without any surgical intervention in the interval. Only hemispheres without a prior revascularization procedure were considered.

\section{Neurological Assessment}

A neurologist (L.J.) retrospectively reviewed patient symptomatology derived from the electronic medical record. Symptomatic hemispheres were defined as those with either a history of recurrent localizable transient ischemic attacks or persistent neurological deficits (motor, sensory, and/or language) referable to the hemisphere. Psychological symptoms, deficits in concentration and memory, and/or headache were not included, given the potential ambiguity in localization.

\section{Imaging Protocol}

The BOLD imaging sequences consisted of a T2*weighted single-shot gradient-recalled echo planar imaging acquisition (slice thickness $5 \mathrm{~mm}$, TR/TE 2000/35 $\mathrm{msec}$, field of view $240 \times 240 \mathrm{~mm}$, spatial resolution 3 $\times 3 \times 5 \mathrm{~mm}$ ) across the entire brain. The experimental paradigm consisted of 5 total blocks each of 3 minutes' duration, beginning and ending with the delivery of medical-grade (purified) room air and interleaved with hypercapnic gas $\left(5 \% \mathrm{CO}_{2} / 95 \% \mathrm{O}_{2}\right)$ administration. We have recently quantified relationships between such hypercapnic hyperoxic and hypercapnic normoxic stimuli in healthy adults and patients with intracranial stenosis, ${ }^{9}$ and have demonstrated the ability of this stimulus to be performed safely in a large volume of patients, to provide contrast consistent with symptomatology and lateralizing disease, and to correlate with perfusion reactivity on appropriate postprocessing. Gas delivery was performed using a custom-made nonrebreathing face mask, and core physiological parameters including end-tidal $\mathrm{CO}_{2}\left(\mathrm{ETCO}_{2}\right)$, heart rate, blood pressure, and arterial oxygen saturation were monitored throughout the experiment.

\section{Radiological Evaluation}

\section{Prior Infarct}

Each hemisphere under review was considered separately. Two board-certified neuroradiologists (M.K.S., L.T.D.) who were blinded to clinical history and hemodynamic findings reviewed FLAIR imaging acquired at the time of BOLD MRI to determine the presence of infarct. The T2-weighted imaging was also reviewed when available. For lacunar infarcts, a size criterion for hyperintense lesions of greatest axial diameter $\geq 4 \mathrm{~mm}$ on T2-weighted imaging was used to separate prior infarcts from white matter changes. ${ }^{16,22}$ The T1-weighted sequence was used to verify encephalomalacia when infarcts were suspected based on the FLAIR sequence.

\section{Cerebrovascular Reactivity}

Healthy control volunteers $(n=11$; age $46 \pm 12$ years [mean $\pm \mathrm{SD}])$ and patients $(\mathrm{n}=25 ; 42 \pm 13.5$ years $)$ underwent structural (T1-weighted, T2-weighted, FLAIR, MR angiography) and hemodynamic (T2*- and CBF-weighted) 3-T MRI (Fig. 1). The ICA territory was defined by a predetermined mask (Fig. 2). The ICA-territory CVR was assessed using susceptibility-weighted MRI during a hypercapnic $\left(\triangle \mathrm{ETCO}_{2}\right.$ approximately $5 \mathrm{~mm} \mathrm{Hg} ; 2$ repeats) stimulus and normalized to cerebellar CVR. For each patient hemisphere (anterior circulation), the number of standard deviations by which CVR differed from the control mean CVR (Z-statistic: mean $=0.69, \mathrm{SD}=0.19$ ) was calculated.

\section{Digital Subtraction Angiography}

Moyamoya changes on DSA were scored with a modified Suzuki score (mSS), ranging from 0 to IV by 2 neuroradiologists (M.K.S., L.T.D., Table 1), with higher grades representing more severe disease. ${ }^{30}$ The mSS accounts for ICA, middle cerebral artery (MCA), and anterior cerebral artery disease, along with the presence or absence 

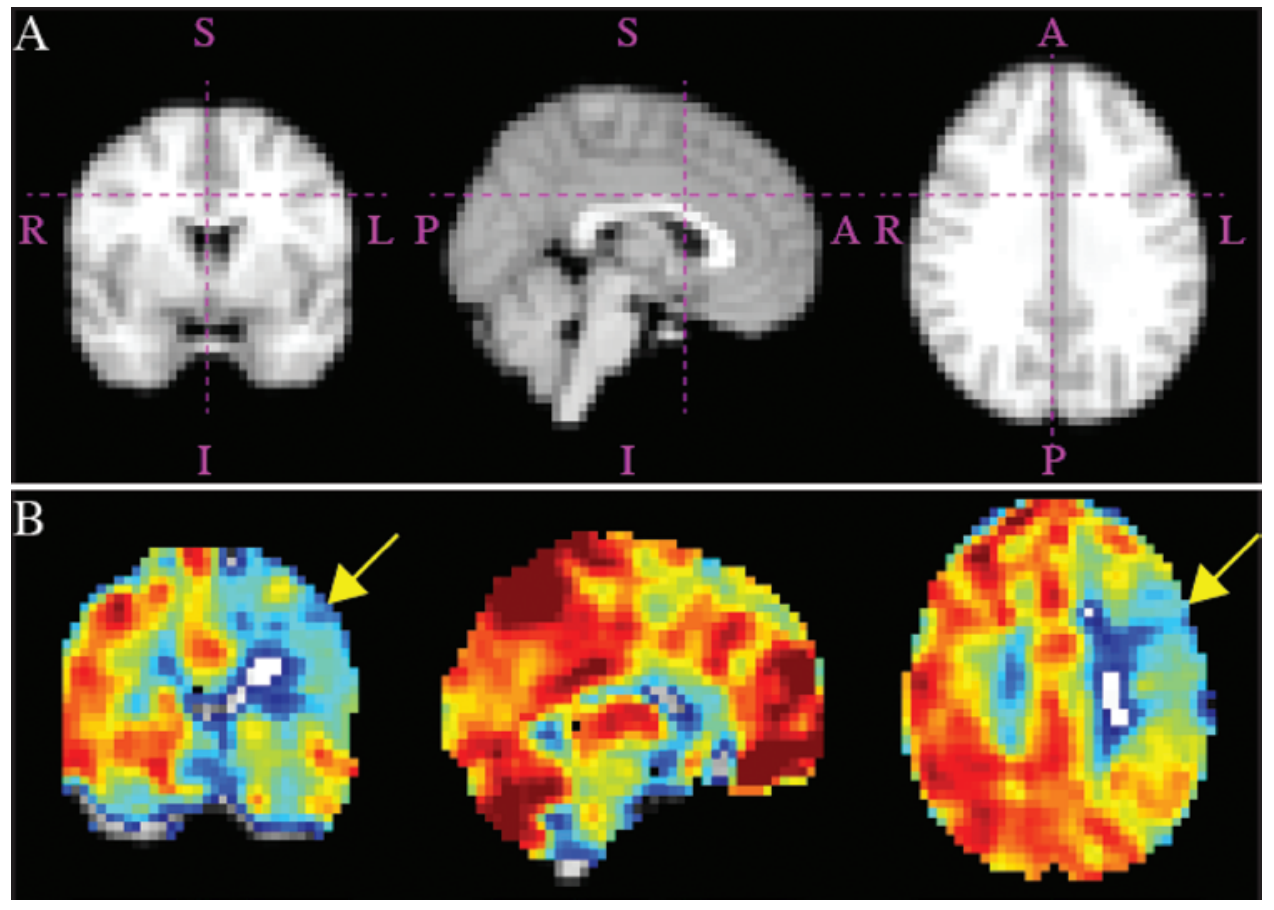

FIG. 1. Admission MRI studies obtained in a patient with a symptomatic left hemisphere. Corresponding atlas maps for hemodynamic sections (A) and orthogonal representations of reactivity maps (B), demonstrating impairment in CVR in the left hemisphere (yellow arrows). Right hemisphere (asymptomatic) PIRAMD score: 0 (Grade 1); left hemisphere (symptomatic) PIRAMD score: 10 (Grade 3). $A$ = anterior; $I=$ inferior; $L=$ left; $P=$ posterior; $R=$ right; $S=$ superior. Figure is available in color online only.

of lenticulostriate collaterals. Regional collateralization on DSA was assessed via modification of a previously reported technique..$^{18,33}$ In brief, DSA was divided into 7 anatomical sites based on Alberta Stroke Program Early
CT Score (ASPECTS)-defined regional vascular territories: ${ }^{28,33}$ M1-M6 and basal ganglia (Fig. 3). An interventional neurologist (M.T.F.) and a neuroradiologist (M.K.S.) working in tandem reviewed each territory on DSA for

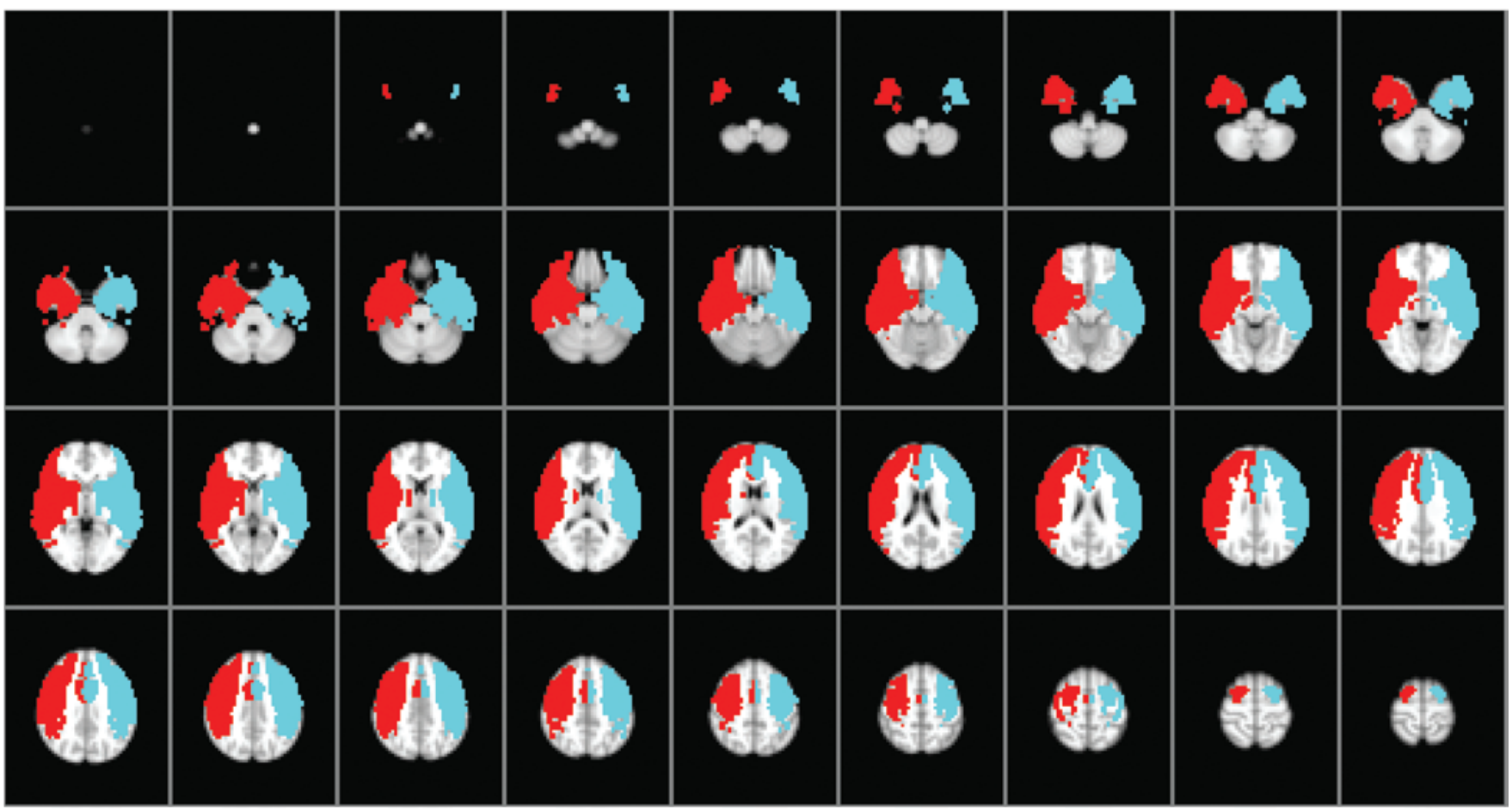

FIG. 2. The ICA territory masks used to define right (red) and left (blue) regions of interest for assessment of CVR. Figure is available in color online only. 
TABLE 1. Modified Suzuki scoring*

\begin{tabular}{|c|c|}
\hline Score & Description of Classification \\
\hline 0 & No evidence of dise \\
\hline I & $\begin{array}{l}\text { Mild-to-moderate stenosis around ICA bifurcation w/ absent or } \\
\text { slightly developed ICA MMD }\end{array}$ \\
\hline$\|$ & $\begin{array}{l}\text { Severe stenosis around the ICA bifurcation or occlusion of } \\
\text { either proximal anterior or MCA branches w/ well-developed } \\
\text { ICA MMD }\end{array}$ \\
\hline III & $\begin{array}{l}\text { Occlusion of both anterior \& MCA branches w/ well-developed } \\
\text { ICA MMD (only a few of anterior or MCA branches or both } \\
\text { are faintly opacified in antegrade fashion through meshwork } \\
\text { of ICA MMD) }\end{array}$ \\
\hline IV & $\begin{array}{l}\text { cclusion of both anterior \& MCA branches w/ } \\
\text { r small amount of ICA MMD (w/o opacification of } \\
\text { terior or MCA branches in antegrade fashion) }\end{array}$ \\
\hline \multicolumn{2}{|c|}{$\begin{array}{l}\text { MMD = moyamoya disease. } \\
\text { * Reprinted with permission from Strother MK, Anderson MD, Singer RJ, Du } \\
\text { L, Moore RD, Shyr Y, et al: Cerebrovascular collaterals correlate with disease } \\
\text { severity in adult North American patients with Moyamoya disease. AJNR Am J } \\
\text { Neuroradiol 35:1318-1324, 2014. }\end{array}$} \\
\hline
\end{tabular}

each patient and assessed whether or not regional $\mathrm{CBF}$ was impaired for the ASPECTS-defined regions. A territory was considered impaired if there were no visible collateral vessels supplying the ischemic site or if there were collaterals only to the periphery of the ischemic site. A territory was considered not to be impaired if collateral flow provided complete irrigation of the ischemic bed or if there was normal anterograde flow. Flow classification
TABLE 2. The PIRAMD scoring system

\begin{tabular}{llc}
\hline Variable & \multicolumn{1}{c}{ Characteristics } & Points \\
\hline MRI & Prior infarct & 1 \\
\hline CVR & Decreased & 3 \\
\hline mSS & $\geq$ Grade II & 3 \\
\hline Collaterals & $\geq 2$ territories impaired & 3 \\
\hline
\end{tabular}

was made based on consensus. The total number of impaired territories (0-7) was counted for each hemisphere.

\section{Optimization of PIRAMD System and Analysis}

Each component of PIRAMD was converted to a categorical variable and given a preliminary, simplified scoring system. The preliminary scoring system was optimized via simple logistic regression analysis and receiver operating characteristic (ROC) curve analysis by using symptomatology as the dependent variable to determine a clinically valid and statistically significant stratification system. Acceptability criteria were $\mathrm{p}<0.05$ for binary logistic regression analysis, and area under the curve (AUC) $>0.6$ for ROC curve analysis. The PIRAMD component grading is summarized in Table 2 .

\section{Grading With the PIRAMD System}

The relative weight for each factor in the PIRAMD score was determined by using the factor's odds ratio from simple logistic regression analysis. The lowest odds ratio was used as the baseline by which all other factors
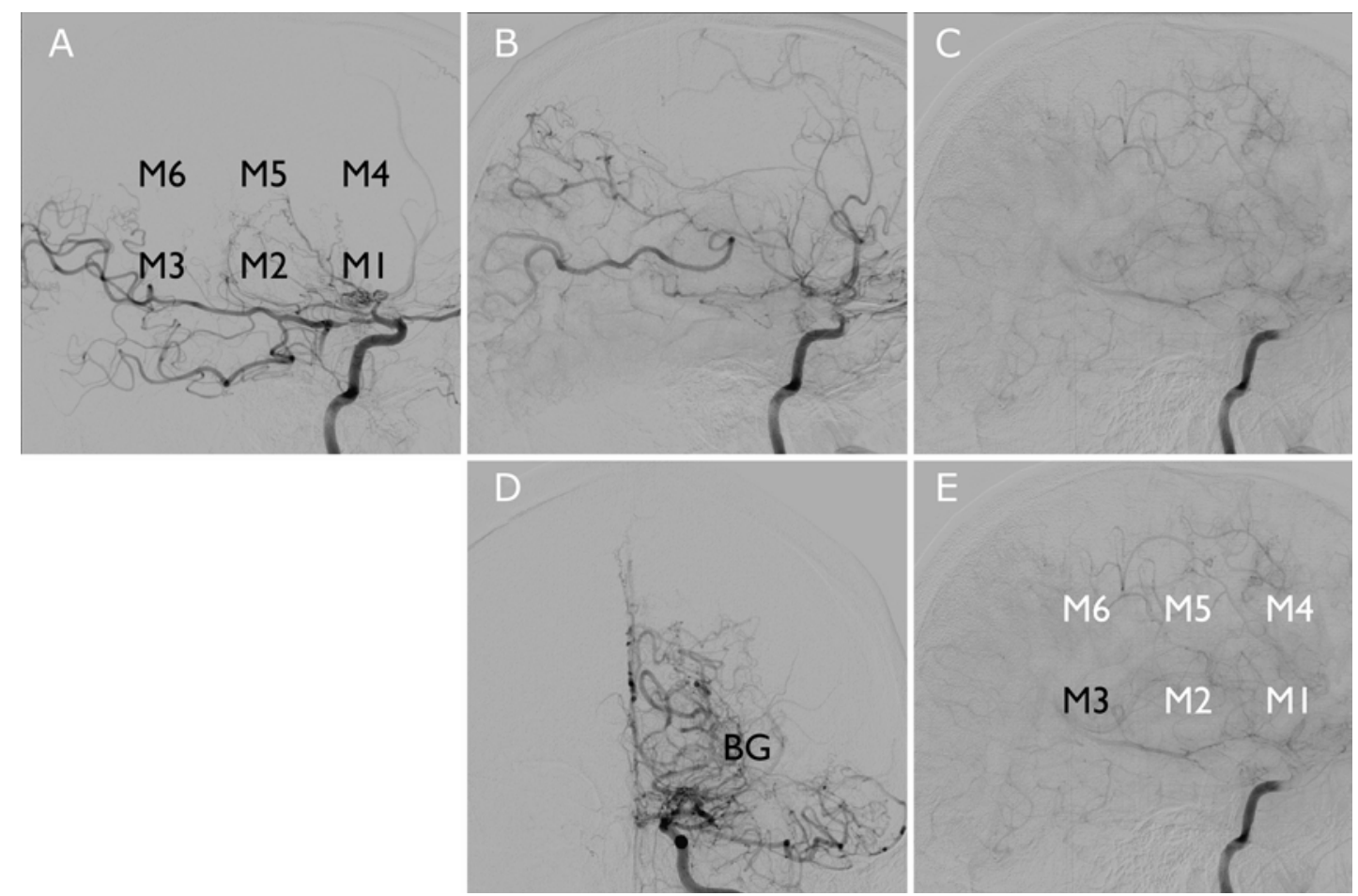

FIG. 3. Lateral projections (A, early-; B, mid-; and C, delayed-phase sequences) from left ICA injection on DSA. The 7 DSA territories measured are labeled in the lower row from the same left ICA injection (D, AP projection; E, lateral projection); impaired regions with delayed perfusion from collaterals are labeled in white $(E) . B G=$ basal ganglia; $M 1-M 6=A S P E C T S$ territories. 
were scaled. The weighted scores for each component of PIRAMD were added together for a raw PIRAMD score. The PIRAMD score was divided into 3 grades: PIRAMD 1, PIRAMD 2, and PIRAMD 3. Grade stratification was determined through optimization by ROC curve analysis and simple binary logistic regression analysis, considering PIRAMD score as a discrete independent variable for those purposes. The ROC and binary logistic regression analyses were also repeated for PIRAMD grade. Acceptability criteria were $\mathrm{p}<0.01$ for binary logistic regression analysis, and AUC $>0.8$ for ROC curve analysis.

\section{Validation of the PIRAMD System}

Additional internal validation was conducted on the data set via a bootstrap method. The $95 \%$ confidence interval estimates for the binary logistic regression analyses were generated in SPSS using a bootstrap sample size of 1000. Bootstrapped $p$ values and confidence intervals were obtained and reported. The ROC bootstrapping also was performed in SPSS using an adapted public domain macro (available at http://gjyp.nl/marta/ [Accessed February 16, 2016]), with a bootstrap sample size of 1000 . Bootstrapped AUC and 95\% confidence intervals were obtained and reported.

\section{Results}

\section{Participants in Study}

There were 25 participants with moyamoya in the study, accounting for 46 hemispheres (Table 3). The mean age was 42 years, with an SD of 12 years. Most patients were female $(20 ; 80 \%)$. There were $28(60.9 \%)$ symptomatic hemispheres. The majority of patients had bilateral disease $(22 ; 88 \%)$. Four of the 46 hemispheres analyzed $(8.7 \%)$ had undergone a prior contralateral revascularization surgery, but none had a prior ipsilateral revascularization surgery. The mean interval between MRI scan and diagnostic angiography was 30 days, with an SD of 24.2 days.

\section{Structural MRI Data}

Thirty (65.2\%) hemispheres had a prior infarct on T2weighted FLAIR imaging. With a prior infarct, the odds of having a symptomatic hemisphere were 4.6 times greater than without a prior infarct; however, the bootstrapped confidence interval estimates crossed unity and were not statistically significant $(95 \%$ CI $0.3-3.3, \mathrm{p}=0.016$; Table 4). The AUC for prior infarct was 0.670 (95\% CI $0.539-$ $0.802)$.

\section{Hemodynamic MRI Data}

After normalizing ICA territory CVR by total cerebellar CVR, 35 (76.1\%) hemispheres had reduced normalized CVR relative to the control cohort. With CVR lower than control CVR, the odds of having a symptomatic hemisphere were 13 times greater than when normal or increased CVR was present (95\% CI 1.1-22.6, $\mathrm{p}=0.002)$. The AUC for CVR was 0.716 (95\% CI 0.585-0.844).

\section{Angiographic Data}

Thirty-eight hemispheres (82.6\%) had an mSS of $\geq$
TABLE 3. Characteristics of patients and hemispheres in study

\begin{tabular}{|c|c|}
\hline Characteristic & Value \\
\hline \multicolumn{2}{|l|}{ By patient $(n=25)$} \\
\hline \multicolumn{2}{|l|}{ Demographic data } \\
\hline Age in yrs, mean $\pm S D$ & $42 \pm 13.5$ \\
\hline Female sex & $20(80 \%)$ \\
\hline \multicolumn{2}{|l|}{ Race } \\
\hline Asian/Pacific islander & $3(12 \%)$ \\
\hline Black/African American & $8(32 \%)$ \\
\hline White/Caucasian, Hispanic/Latino & $1(4 \%)$ \\
\hline White/Caucasian, non-Hispanic/Latino & $13(52 \%)$ \\
\hline \multicolumn{2}{|l|}{ Clinical data } \\
\hline Bilat moyamoya & $22(88 \%)$ \\
\hline Days btwn MRI \& DSA, mean \pm SD & $30 \pm 24.2$ \\
\hline \multicolumn{2}{|l|}{ By hemisphere $(n=46)$} \\
\hline \multicolumn{2}{|l|}{ Clinical data } \\
\hline Symptomatic & $28(60.9 \%)$ \\
\hline Prior contralat revascularization & $4(8.7 \%)$ \\
\hline \multicolumn{2}{|l|}{ Infarct } \\
\hline No prior infarct & $16(34.8 \%)$ \\
\hline Prior infarct & $30(65.2 \%)$ \\
\hline \multicolumn{2}{|l|}{ CVR } \\
\hline Normal/increased & $11(23.9 \%)$ \\
\hline Decreased & $35(76.1 \%)$ \\
\hline \multicolumn{2}{|l|}{$\mathrm{mSS}$} \\
\hline 0 & $3(6.5 \%)$ \\
\hline I & $5(10.9 \%)$ \\
\hline ॥ & $20(43.5 \%)$ \\
\hline III & $14(30.4 \%)$ \\
\hline IV & $4(8.7 \%)$ \\
\hline \multicolumn{2}{|l|}{ No. of collaterals impaired } \\
\hline 0 & $10(21.7 \%)$ \\
\hline 1 & $1(2.2 \%)$ \\
\hline 2 & $6(13.0 \%)$ \\
\hline 3 & $10(21.7 \%)$ \\
\hline 4 & $10(21.7 \%)$ \\
\hline 5 & $3(6.5 \%)$ \\
\hline 6 & $3(6.5 \%)$ \\
\hline 7 & $3(6.5 \%)$ \\
\hline \multicolumn{2}{|l|}{ PIRAMD grade } \\
\hline 1 & $8(17.4 \%)$ \\
\hline 2 & $18(39.1 \%)$ \\
\hline 3 & $20(43.5 \%)$ \\
\hline
\end{tabular}

Grade II. With an mSS in this range, the odds of having a symptomatic hemisphere were 17.2 times greater than with Grade 0-I mSS (95\% CI 1.1-22.7, $\mathrm{p}=0.008$ ). The AUC for mSS was 0.678 (95\% CI 0.560-0.793).

With regard to collateral flow impairment, 35 (76.1\%) hemispheres had 2-7 territories impaired. With $\geq 2 \mathrm{im}-$ paired territories, the odds of having a symptomatic hemisphere were 13 times greater than with $0-1$ impaired ter- 
TABLE 4. Correlations with symptomatology

\begin{tabular}{lllllrl}
\hline \multicolumn{1}{c}{ Component } & AUC & $95 \% \mathrm{Cl}$ & Subscore & OR & $95 \% \mathrm{Cl}$ & $\mathrm{pValue}$ \\
\hline Prior infarct & 0.670 & $0.539-0.802$ & Present & 4.6 & $0.3-3.3$ & 0.016 \\
\hline CVR & 0.716 & $0.585-0.844$ & Decreased & 13.0 & $1.1-22.6$ & 0.002 \\
\hline mSS & 0.678 & $0.560-0.793$ & $\geq$ Grade II & 17.2 & $1.1-22.7$ & 0.008 \\
\hline Collaterals & 0.713 & $0.593-0.836$ & $\geq 2$ territories & 13.0 & $1.1-22.7$ & 0.006 \\
\hline PIRAMD Grade & 0.845 & $0.735-0.956$ & 2 & NA & $20.4-22.5$ & 0.004 \\
& & & 3 & NA & $22.3-42.4$ & 0.004 \\
\hline
\end{tabular}

NA = not applicable.

ritories (95\% CI 1.1-22.7, $\mathrm{p}=0.006)$. The AUC for collaterals was 0.713 (95\% CI $0.593-0.836$ ).

\section{Development of PIRAMD Score}

After the simple analysis of the individual PIRAMD factors, the relative weighting for each factor was determined by scaling the odds ratio for prior infarct (4.6), because this had the lowest odds ratio. The PIRAMD scoring system is summarized in Table 2. Scores were added to ascertain the hemisphere's PIRAMD score, and in this way, PIRAMD was scored from 0 to 10 , with increasing score representing increasing impairment (Fig. 4).

\section{The PIRAMD Grade}

Hemispheres were divided into 3 severity grades based on total PIRAMD score: Grade 1, 0-5 points; Grade 2, 6-9 points; and Grade 3, 10 points. There were 0/8 (0\%), 10/18 (55.6\%), and 18/20 (90\%) symptomatic PIRAMD Grade 1, 2, and 3 hemispheres, respectively (Fig. 5). The AUC for the PIRAMD grade (Grade 1-3) was 0.845 (95\% CI 0.735-0.956). The AUC for the PIRAMD score (i.e., 0-10) was 0.860 (95\% CI 0.746-0.974).

\section{Discussion}

The PIRAMD classification is a simple scoring system for impairment in moyamoya, which uses noninvasive functional MRI parameters in addition to angiographic data. The PIRAMD score was found to correlate well with symptomatology (AUC 0.860). There were 0/8 (0\%), 10/18 (55.6\%), and 18/20 (90\%) symptomatic PIRAMD Grade 1, 2 , and 3 hemispheres, respectively.

Patients with moyamoya are heterogeneous with regard to their clinical presentations and outcomes; however, angiographic studies alone may not be sufficient to understand patient pathophysiology. Clinical severity does not follow a perfect correlation with angiography, because proximal occlusions may be completely compensated by robust pial and lenticulostriate autocollateralization. In contrast, uncompensated mild stenosis may portend a severe course. ${ }^{20,30}$ In some cases, angiography findings may not even correlate with hemodynamic impairment. ${ }^{4}$

Because surgical candidacy is weighted heavily by imaging appearance and symptomatology, efforts to stratify patients for intervention are critical. A contemporary issue in the management of patients with moyamoya is the selection of impaired individuals who are likely to benefit from surgical revascularization. Cerebrovascular reactivity may be predictive of outcome and may be useful in noninvasive monitoring of such patients..$^{14,19,25,27}$ Han et al. have shown that postrevascularization CVR correlates with graft patency and clinical outcomes in moyamoya. ${ }^{14}$

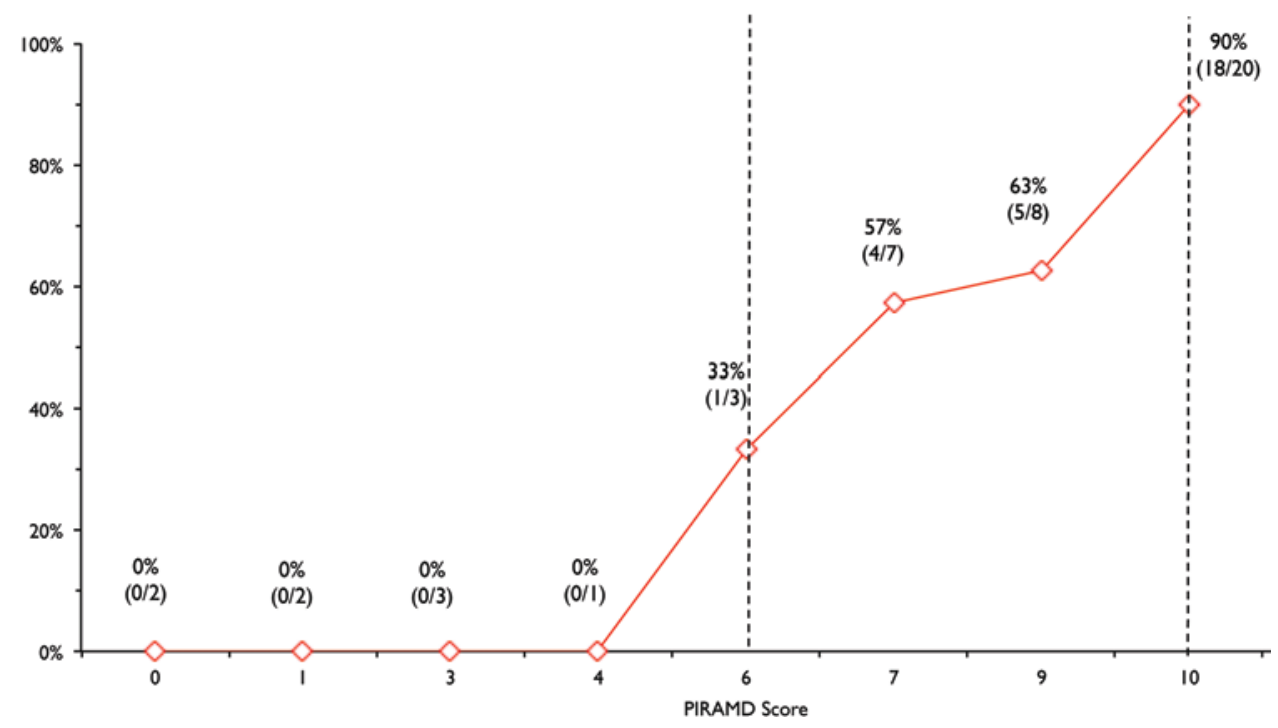

FIG. 4. Graph showing the PIRAMD grade versus proportion of patients who were symptomatic. Vertical dashed lines represent PIRAMD Grade $2(\geq 6)$ and Grade $3(10)$ demarcations, respectively. Figure is available in color online only. 


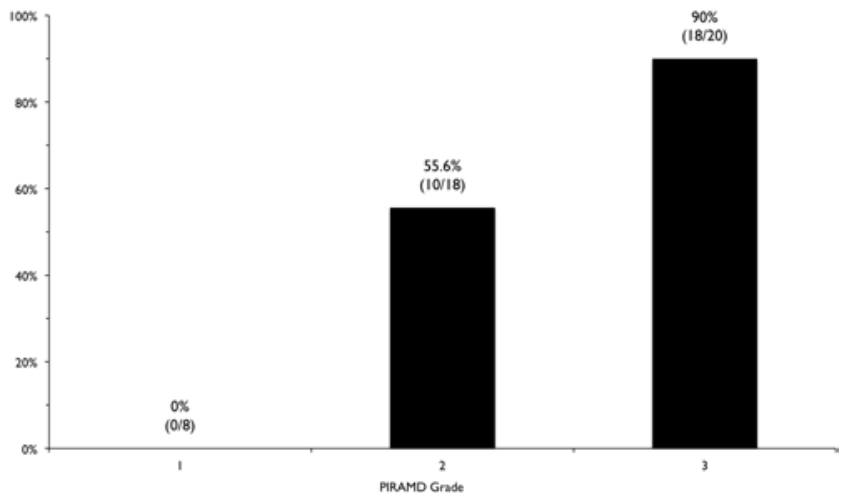

FIG. 5. Bar graph showing the PIRAMD grade versus symptomatology. There were $0 / 8(0 \%), 10 / 18(55.6 \%)$, and $18 / 20(90 \%)$ symptomatic PIRAMD Grade 1, 2, and 3 hemispheres, respectively.

In intracranial stenosis in general, Mandell et al. showed that patients with impaired CVR were more likely to have hemodynamic normalization after extracranial-intracranial revascularization. ${ }^{24}$

There has been a robust effort in the field to quantify and understand hemodynamic impairment in moyamoya. The CBF and CBV increase in the early stages of impairment, and oxygen extraction fraction increases when $\mathrm{CBF}$ cannot increase sufficiently to meet oxygen demands. ${ }^{21}$ The current methods available for assessing these impairments include PET, SPECT, xenon-enhanced CT (Xe-CT), dynamic perfusion CT, dynamic susceptibility contrast MRI, arterial spin labeling MRI, and Doppler ultrasound (see Table 1 in Lee et al. ${ }^{21}$ ). However, many of these methods (e.g., $\mathrm{PET}$, SPECT, Xe-CT, and dynamic perfusion $\mathrm{CT}$ ) require ionizing radiation exposure and/or administration of exogenous contrast (e.g., $\mathrm{Gd}$, which causes renal failure in up to $2 \%$ of $\operatorname{cases}^{3}$ ). Diagnostic angiography carries risk, with a complication rate as high as $1.2 \%$ in the Asymptomatic Carotid Atherosclerosis Study (ACAS), ${ }^{32}$ in addition to potential dose-dependent radiation-induced skin injuries. ${ }^{31}$

Magnetic resonance imaging can use blood oxygenation level as an endogenous contrast agent and therefore does not require exogenous contrast or ionizing radiation. It can be acquired serially during routine structural MRI and therefore holds promise as a noninvasive, readily available, and valid adjunct to routine imaging of patients with moyamoya. Additionally, MR offers improved spatial (3to 5-mm isotropic) and temporal (2- to 3-second) resolution, and may be more clinically available compared with PET and SPECT, especially in nonspecialized hospitals.

In patients with intracranial disease, compensation for secondary reductions in cerebral perfusion pressure may initially be achieved via an increase in CBV and CBF., To assess this autoregulatory capacity, a vasostimulus such as carbogen can be administered. Carbogen serves to increase the arterial partial pressure of $\mathrm{O}_{2}$ and $\mathrm{CO}_{2}, \mathrm{CBF}$, and $\mathrm{CBV}$, and in turn increases blood oxygenation. The resulting increase in the ratio of oxyhemoglobin compared with deoxyhemoglobin will lead to an increase in T2*-weighted MRI signal. The magnitude of this change in the BOLD signal, or CVR, reflects the ability of vessels to regulate $\mathrm{CBF}$ and $\mathrm{CBV}$, indicating how close the parenchyma is to failing to meet the hemodynamic demand. This permits an endogenous signal to be measured, rather than having to rely on exogenous contrasts or acetazolamide. Carbogen is a safe substance for CVR measurement; in our experience with 92 consecutive patients, carbogen elicited no shortterm neurological events, and longer-term (2-year) events fell within the expected range for patients with intracranial stenosis. ${ }^{9}$

Although the role of CVR in predicting long-term stroke risk is not yet completely known, numerous studies have established a strong correlation between CVR and intracranial vascular disease., ${ }^{9,15}$ In patients with moyamoya, strong inverse relationships between mean CVR and both the Suzuki score and the presence of collateral vessels have been identified. ${ }^{4,17}$ Patients with moyamoya that is refractory to medical management who undergo surgical revascularization have been shown to demonstrate postsurgical revascularization improvements in CVR in regions that were previously compromised.14,23,24

Using Xe-CT with acetazolamide challenge in 40 patients (80 hemispheres), Czabanka et al. created a similar scoring mechanism for moyamoya severity. ${ }^{6}$ Compromised cerebrovascular reserve capacity was defined with $\mathrm{Xe}-\mathrm{CT}$ as a CBF decrease greater than 5\% after acetazolamide challenge. Although for methodological reasons we were unable to compare PIRAMD to the Czabanka system directly, the AUC was similar between populations (0.80 for the Czabanka score vs 0.845 for the PIRAMD grade). Although that study has laid the foundation for using CVR in moyamoya severity stratification, the technical innovations that have occurred since then have led us to conclude that PIRAMD might be a more favorable scoring system for patients. This is particularly true in the US, where the use of xenon is not widespread due to concerns in the literature and from the FDA related to reports of respiratory side effects associated with xenon. ${ }^{5}$ Symptomatic classification was more conservative in our study. Whereas Czabanka et al. considered psychological or headache symptoms as bilaterally symptomatic, these were not counted in our study due to difficulty in localization. This may account for the apparent difference in the prevalence of symptomatic hemispheres between cohorts $(60.9 \%$ in our study, $68 \%$ in the study by Czabanka et al.).

We find it interesting that a milder correlation with symptomatology in our study was found with prior infarct. This is not to say that prior infarct is not important; it indicates that significant hemodynamic impairment has already occurred. In fact, we found post hoc that $22 / 28$ (78.6\%) of symptomatic hemispheres had an infarct. There was, however, a high rate of clinically silent infarcts $(8 / 30$ infarcts, $26.7 \%$ ), particularly in the watershed distribution. There was considerable collinearity with other PIRAMD components; if a prior infarct was present, impairment in another PIRAMD component was also present in all but 2 cases $(28 / 30 ; 93.3 \%)$. Unsurprisingly, with prior infarct the mean PIRAMD score was 8.5 , versus 6.2 without ( $p$ $=0.013$ ). However, for the hemispheres without infarct (especially the $21 \%$ of symptomatic hemispheres without infarct), the PIRAMD score adds more clarity to hemodynamic impairment than infarct assessment alone. Patients without infarct may have other impairments that increase 
the PIRAMD score, and this may weigh more heavily on the decision to revascularize.

Therefore, it is useful to complement this information with functional measures of impairment. The angiographic assessment of collateral perfusion is a strong contributor to the PIRAMD score, because it allows a functional, dynamic assessment of territory perfusion. However, it is invasive and requires radiation, which may limit its use in serial monitoring. Thus, we offer a functional complement of parenchymal impairment via BOLD MRI. ${ }^{8}$ The measurement of CVR using MRI provides a noninvasive method for assessing hemodynamic instability. We found that reduced CVR carried a 13-fold increase in the odds of the patient being symptomatic. When synthesizing angiographic and structural/hemodynamic MRI evidence of impairment via the PIRAMD score, the probability of being symptomatic can be calculated. We found that hemispheres were asymptomatic until the PIRAMD score reached 6 , at which point a precipitous proportional rise in symptomatology occurred (Fig. 4).

The PIRAMD severity grades may be useful in counseling patients for further management. In particular, patients with PIRAMD Grade 3, if not already symptomatic, might have a high risk of becoming symptomatic, and surgery should be more strongly considered. In contrast, patients with PIRAMD Grade 1 might not become symptomatic, and conservative management with serial monitoring may be recommended. Of course, although reduced CVR may be associated with greater stroke risk,,$^{13}$ the long-term risks of PIRAMD grades have not yet been evaluated, and would need to be assessed in larger prospective studies. Nonetheless, the strong correlation between PIRAMD score and symptomatology suggests it may still have a role in patient stratification.

\section{Limitations of the Study}

This study is limited by its retrospective nature and small sample size, necessitating univariate analysis. Although we included patients if DSA was performed within 90 days, it would have been more ideal to have these studies obtained concurrently; the mean interval between angiography and MRI was 30 days. Although we performed a limited internal validation via bootstrapping, prospective external validation with a larger number of patients is needed. This study addresses historic symptom risk, and does not predict future symptoms or postoperative response, and therefore we are continuing to evaluate this prospectively.

\section{Conclusions}

A scoring system for total impairment in moyamoya is proposed in which noninvasive hemodynamic and structural MRI parameters are used, along with conventional angiography. This scoring system was found to correlate with symptomatology and may provide a measure of hemodynamic severity in moyamoya, which could be used for guiding management decisions and evaluating intervention response.

\section{Acknowledgments}

This work was supported by the National Institutes of Health
(5R01NS078828-02). Mr. Ladner received funding via the Medical Student Summer Research Fellowship from the AANS.

\section{References}

1. Arteaga DF, Strother MK, Faraco CC, Jordan LC, Ladner TR, Dethrage LM, et al: The vascular steal phenomenon is an incomplete contributor to negative cerebrovascular reactivity in patients with symptomatic intracranial stenosis. J Cereb Blood Flow Metab 34:1453-1462, 2014

2. Blicher JU, Stagg CJ, O'Shea J, Østergaard L, MacIntosh BJ, Johansen-Berg H, et al: Visualization of altered neurovascular coupling in chronic stroke patients using multimodal functional MRI. J Cereb Blood Flow Metab 32:2044-2054, 2012

3. Briguori C, Colombo A, Airoldi F, Melzi G, Michev I, Carlino M, et al: Gadolinium-based contrast agents and nephrotoxicity in patients undergoing coronary artery procedures. Catheter Cardiovasc Interv 67:175-180, 2006

4. Calamante F, Ganesan V, Kirkham FJ, Jan W, Chong WK, Gadian DG, et al: MR perfusion imaging in moyamoya syndrome: potential implications for clinical evaluation of occlusive cerebrovascular disease. Stroke 32:2810-2816, 2001

5. Carlson AP, Brown AM, Zager E, Uchino K, Marks MP, Robertson C, et al: Xenon-enhanced cerebral blood flow at $28 \%$ xenon provides uniquely safe access to quantitative, clinically useful cerebral blood flow information: a multicenter study. AJNR Am J Neuroradiol 32:1315-1320, 2011

6. Czabanka M, Peña-Tapia P, Schubert GA, Heppner FL, Martus $\mathrm{P}$, Horn P, et al: Proposal for a new grading of Moyamoya disease in adult patients. Cerebrovasc Dis 32:41-50, 2011

7. Derdeyn CP, Videen TO, Yundt KD, Fritsch SM, Carpenter DA, Grubb RL, et al: Variability of cerebral blood volume and oxygen extraction: stages of cerebral haemodynamic impairment revisited. Brain 125:595-607, 2002

8. Donahue MJ, Ayad M, Moore R, van Osch M, Singer R, Clemmons P, et al: Relationships between hypercarbic reactivity, cerebral blood flow, and arterial circulation times in patients with moyamoya disease. J Magn Reson Imaging 38:1129-1139, 2013

9. Donahue MJ, Dethrage LM, Faraco CC, Jordan LC, Clemmons P, Singer R, et al: Routine clinical evaluation of cerebrovascular reserve capacity using carbogen in patients with intracranial stenosis. Stroke 45:2335-2341, 2014

10. Donahue MJ, Strother MK, Hendrikse J: Novel MRI approaches for assessing cerebral hemodynamics in ischemic cerebrovascular disease. Stroke 43:903-915, 2012

11. Faraco CC, Strother MK, Dethrage LM, Jordan L, Singer R, Clemmons PF, et al: Dual echo vessel-encoded ASL for simultaneous BOLD and CBF reactivity assessment in patients with ischemic cerebrovascular disease. Magn Reson Med 73:1579-1592, 2015

12. Gao YZ, Zhang JJ, Liu H, Wu GY, Xiong L, Shu M: Regional cerebral blood flow and cerebrovascular reactivity in Alzheimer's disease and vascular dementia assessed by arterial spin-labeling magnetic resonance imaging. Curr Neurovasc Res 10:49-53, 2013

13. Gupta A, Chazen JL, Hartman M, Delgado D, Anumula N, Shao H, et al: Cerebrovascular reserve and stroke risk in patients with carotid stenosis or occlusion: a systematic review and meta-analysis. Stroke 43:2884-2891, 2012

14. Han JS, Abou-Hamden A, Mandell DM, Poublanc J, Crawley AP, Fisher JA, et al: Impact of extracranial-intracranial bypass on cerebrovascular reactivity and clinical outcome in patients with symptomatic moyamoya vasculopathy. Stroke 42:3047-3054, 2011

15. Han JS, Mikulis DJ, Mardimae A, Kassner A, Poublanc J, Crawley AP, et al: Measurement of cerebrovascular reactivity in pediatric patients with cerebral vasculopathy using blood oxygen level-dependent MRI. Stroke 42:1261-1269, 2011 
16. Hassan A, Hunt BJ, O'Sullivan M, Parmar K, Bamford JM, Briley D, et al: Markers of endothelial dysfunction in lacunar infarction and ischaemic leukoaraiosis. Brain 126:424-432, 2003

17. Heyn C, Poublanc J, Crawley A, Mandell D, Han JS, Tymianski M, et al: Quantification of cerebrovascular reactivity by blood oxygen level-dependent MR imaging and correlation with conventional angiography in patients with Moyamoya disease. AJNR Am J Neuroradiol 31:862-867, 2010

18. Kim JJ, Fischbein NJ, Lu Y, Pham D, Dillon WP: Regional angiographic grading system for collateral flow: correlation with cerebral infarction in patients with middle cerebral artery occlusion. Stroke 35:1340-1344, 2004

19. Kohno K, Oka Y, Kohno S, Ohta S, Kumon Y, Sakaki S: Cerebral blood flow measurement as an indicator for an indirect revascularization procedure for adult patients with moyamoya disease. Neurosurgery 42:752-758, 1998

20. Kuroda S, Hashimoto N, Yoshimoto T, Iwasaki Y: Radiological findings, clinical course, and outcome in asymptomatic moyamoya disease: results of multicenter survey in Japan. Stroke 38:1430-1435, 2007

21. Lee M, Zaharchuk G, Guzman R, Achrol A, Bell-Stephens T, Steinberg GK: Quantitative hemodynamic studies in moyamoya disease: a review. Neurosurg Focus 26(4):E5, 2009

22. Longstreth WT Jr, Bernick C, Manolio TA, Bryan N, Jungreis CA, Price TR: Lacunar infarcts defined by magnetic resonance imaging of 3660 elderly people: the Cardiovascular Health Study. Arch Neurol 55:1217-1225, 1998

23. Ma Y, Li M, Jiao LQ, Zhang HQ, Ling F: Contralateral cerebral hemodynamic changes after unilateral direct revascularization in patients with moyamoya disease. Neurosurg Rev 34:347-354, 2011

24. Mandell DM, Han JS, Poublanc J, Crawley AP, Fierstra J, Tymianski M, et al: Quantitative measurement of cerebrovascular reactivity by blood oxygen level-dependent MR imaging in patients with intracranial stenosis: preoperative cerebrovascular reactivity predicts the effect of extracranialintracranial bypass surgery. AJNR Am J Neuroradiol 32:721-727, 2011

25. Mandell DM, Han JS, Poublanc J, Crawley AP, Stainsby JA, Fisher JA, et al: Mapping cerebrovascular reactivity using blood oxygen level-dependent MRI in Patients with arterial steno-occlusive disease: comparison with arterial spin labeling MRI. Stroke 39:2021-2028, 2008

26. Markus H, Cullinane M: Severely impaired cerebrovascular reactivity predicts stroke and TIA risk in patients with carotid artery stenosis and occlusion. Brain 124:457-467, 2001

27. Mikulis DJ, Krolczyk G, Desal H, Logan W, Deveber G, Dirks P, et al: Preoperative and postoperative mapping of cerebrovascular reactivity in moyamoya disease by using blood oxygen level-dependent magnetic resonance imaging. J Neurosurg 103:347-355, 2005

28. Pexman JHW, Barber PA, Hill MD, Sevick RJ, Demchuk AM, Hudon ME, et al: Use of the Alberta Stroke Program
Early CT Score (ASPECTS) for assessing CT scans in patients with acute stroke. AJNR Am J Neuroradiol 22:15341542,2001

29. Siero JCW, Hartkamp NS, Donahue MJ, Harteveld AA, Compter A, Petersen ET, et al: Neuronal activation induced BOLD and CBF responses upon acetazolamide administration in patients with steno-occlusive artery disease. Neuroimage 105: 276-285, 2015

30. Strother MK, Anderson MD, Singer RJ, Du L, Moore RD, Shyr Y, et al: Cerebrovascular collaterals correlate with disease severity in adult North American patients with Moyamoya disease. AJNR Am J Neuroradiol 35:1318-1324, 2014

31. Vano E, Fernandez JM, Sanchez RM, Martinez D, Ibor LL, Gil A, et al: Patient radiation dose management in the followup of potential skin injuries in neuroradiology. AJNR Am J Neuroradiol 34:277-282, 2013

32. Walker MD, Marler JR, Goldstein M, Grady P, Toole J, Baker W: Endarterectomy for asymptomatic carotid artery stenosis. Executive Committee for the Asymptomatic Carotid Atherosclerosis Study. JAMA 273:1421-1428, 1995

33. Zaharchuk G, Do HM, Marks MP, Rosenberg J, Moseley ME, Steinberg GK: Arterial spin-labeling MRI can identify the presence and intensity of collateral perfusion in patients with moyamoya disease. Stroke 42:2485-2491, 2011

\section{Disclosures}

The authors report no conflict of interest concerning the materials or methods used in this study or the findings specified in this paper.

\section{Author Contributions}

Conception and design: Ladner, Donahue, Strother. Acquisition of data: Ladner, Donahue, Faraco, Roach, Davis, Jordan, Froehler, Strother. Analysis and interpretation of data: all authors. Drafting the article: Ladner. Critically revising the article: all authors. Reviewed submitted version of manuscript: all authors. Approved the final version of the manuscript on behalf of all authors: Ladner. Statistical analysis: Ladner, Faraco. Administrative/technical/material support: Donahue. Study supervision: Donahue, Strother.

\section{Supplemental Information \\ Previous Presentations}

A portion of the findings herein were presented as an oral abstract at the 2015 AANS/CNS Joint Cerebrovascular Section Annual Meeting on February 9, 2015, in Nashville, TN.

\section{Correspondence}

Travis R. Ladner, 1161 21st Ave. S, CCC-1108 MCN, Nashville, TN 37232-2380. email: travis.r.ladner@vanderbilt.edu. 